\title{
Direct Excitation of Propagating Spin Waves by Focused Ultrashort Optical Pulses
}

\author{
Y. Au, ${ }^{1}$ M. Dvornik, ${ }^{1,2}$ T. Davison, ${ }^{1}$ E. Ahmad, ${ }^{1}$ P. S. Keatley, ${ }^{1}$ A. Vansteenkiste, ${ }^{2}$ \\ B. Van Waeyenberge, ${ }^{2}$ and V. V. Kruglyak ${ }^{1}$ \\ ${ }^{1}$ School of Physics, University of Exeter, Stocker Road, Exeter EX4 4QL, United Kingdom \\ ${ }^{2}$ DyNaMat, Department of Solid State Sciences, Ghent University, Krijgslaan 281 S1, 9000 Ghent, Belgium
}

(Received 31 October 2012; published 25 February 2013)

\begin{abstract}
An all-optical experiment long utilized to image phonons excited by ultrashort optical pulses has been applied to a magnetic sample. In addition to circular ripples due to surface acoustic waves, we observe an $X$-shaped pattern formed by propagating spin waves. The emission of spin waves from the optical pulse epicenter in the form of collimated beams is qualitatively reproduced by micromagnetic simulations. We explain the observed pattern in terms of the group velocity distribution of Damon-Eshbach magnetostatic spin waves in the reciprocal space and the wave vector spectrum of the focused ultrafast laser pulse.
\end{abstract}

The recent proliferation of studies on the interaction of femtosecond optical pulses with magnetic materials [1] has been primarily concerned with exploration and understanding of novel types of ultrafast magnetic phase transitions and the associated promise of a new paradigm of high speed magnetic data storage technology [2-13]. Far less attention has been paid to the possibility of using the ultrafast optical excitation to induce magnetization precession and propagating spin waves [14-18]. However, such practice could lead to important applications (at least in the context of fundamental research) in the emerging field of "magnonics" $[19,20]$. An important breakthrough was recently achieved by Satoh et al., who demonstrated alloptical imaging of propagating magnetostatic spin waves of about $100 \mu \mathrm{m}$ wavelength excited by ultrafast optical pulses in a ferromagnetic dielectric [18]. However, despite the importance of magnetic dielectrics and long wavelength magnetostatic spin waves [21], the ultimate goal of magnonics requires that much shorter wavelength spin waves be excited and studied in magnetic thin films and nanostructures $[16,19]$.

In this Letter, we demonstrate that femtosecond optical pulses focused to a diffraction limited spot by a high quality microscope objective are able to excite spin waves at specific locations on the surface of a thin magnetic film. The propagation of the optically excited spin waves is imaged using a setup labeled here as time resolved optically pumped scanning optical microscope (TROPSOM), which has applications beyond the fields of magnonics and optomagnetism. The employed experimental scheme has been utilized to image optically excited propagating phonons [22], which are also observed in our experiments. As compared to the more conventional methods of spin wave excitation by current carrying microstrips [19,21], TROPSOM yields the benefit of broadband point magnonic sources, which could otherwise be only obtained by means of complex nanofabrication [23]. Inspired by the recent demonstrations of spin wave emission by resonant transducers under a uniform microwave field $[24,25]$, one could even imagine building similar devices to enable effective conversion of the femtosecond laser light into a tailored spin wave emission pattern on a magnonic chip. Here, the combination of the point emission of propagating spin waves and their magneto-optical imaging has allowed us to confirm the formation of spin wave caustics as a result of the highly anisotropic magnonic dispersion.

Our experimental observations agree with predictions of analytical calculations of the dispersion of surfaces acoustic waves (SAWs) and of the numerical micromagnetic calculations in which we have included the longitudinal relaxation of the absolute value of the magnetization vector. The corresponding micromagnetic code is a prerequisite of the successful description of our experimental observations, representing in its own right an important development in the field of micromagnetic modeling [26]. Our micromagnetic simulations reveal a possibility of spin wave excitation by a transient in-plane demagnetizing field induced by tightly focused optical pulses, as opposed to earlier pump-probe experiments employing much larger pump spots and relying on triggering the magnetization dynamics by a transient out-of-plane demagnetizing field. Together, the experimental and theoretical advances reported in this Letter constitute a toolbox necessary to unleash the potential of the interdisciplinary field of photomagnonics [20].

Figure 1(a) illustrates the TROPSOM experimental setup. A train of optical pulses of $800 \mathrm{~nm}$ wavelength and (nominally) $150 \mathrm{fs}$ duration is generated at $80 \mathrm{MHz}$ repetition rate (1) by a Ti:Sapphire laser. Following the conventional pump-probe method [1,20], each laser pulse is split into pump and probe parts. A high numerical aperture coverslip corrected microscope objective (2) is used to focus the pump to a diffraction limited spot on the surface of a $50 \mathrm{~nm}$ thick Permalloy film (3) through its substrate comprising a $430 \mu \mathrm{m}$ thick $C$-plane sapphire 


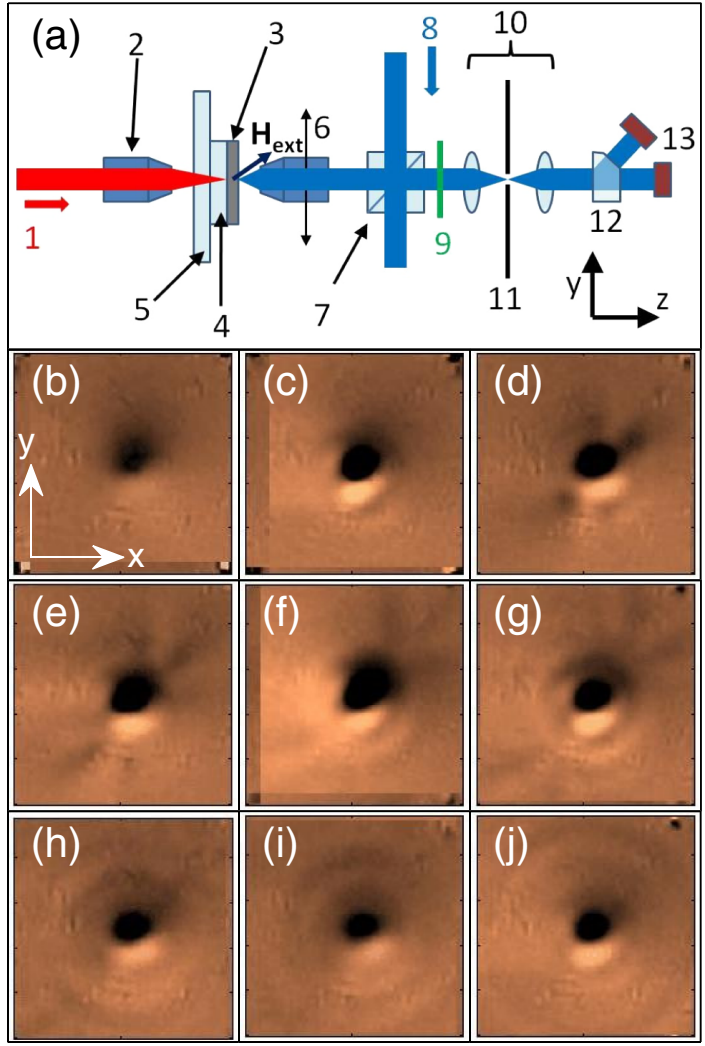

FIG. 1 (color online). (a) Cartoon illustration of the experimental setup: (1) $800 \mathrm{~nm}$ wavelength pump beam, (2) pump beam objective, (3) $50 \mathrm{~nm}$ thick permalloy film, (4) $430 \mu \mathrm{m}$ thick sapphire substrate, (5) $0.17 \mathrm{~mm}$ thick glass coverslip, (6) probe beam objective, (7) nonpolarizing beam splitter, (8) $400 \mathrm{~nm}$ wavelength probe beam, (9) $400 \mathrm{~nm}$ bandpass filter, (10) spatial filter, (11) diaphragm with a $50 \mu \mathrm{m}$ pinhole, (12) polarizing beam splitter, and (13) photodiodes. (b) - (j) Time resolved $10 \times 10 \mu \mathrm{m}^{2}$ Kerr images at time delays of $0.667,1.000,1.200,1.267,1.367,1.450,1.567,1.733$, and $2.017 \mathrm{~ns}$, respectively. The pump-probe time delay of $1 \mathrm{~ns}$ corresponds to the simultaneous arrival of the pump and probe pulses to the sample [27].

plate (4) mounted on a $170 \mu \mathrm{m}$ thick glass coverslip (5). The probe part is converted to a $400 \mathrm{~nm}$ wavelength (8) by a frequency doubling crystal, and after passing through a nonpolarizing 50/50 beam splitter (7) is focused on the opposite surface (with respect to the pump beam) of the Permalloy film by another high quality microscope objective (6), again into a diffraction limited spot. The power of the $800 \mathrm{~nm}$ pump (1) and $400 \mathrm{~nm}$ probe (8) beams is maintained at 60 and $1 \mathrm{~mW}$, respectively. The probe beam objective (6) is mounted on a piezoelectric stage. By scanning the probe objective, we scan the position of the probe spot relative to the pump spot [fixed by the position of the pump objective (2)], thereby enabling the raster imaging of the dynamics excited in the sample by the optical pump. The temporal resolution is obtained by using a retroreflector mounted on a linear translation stage (not shown) to vary the time delay between the arrival of the pump and probe pulses at the sample. A $400 \mathrm{~nm}$ band pass optical filter (9) separates the probe beam from any pump beam component that leaks through the sample. The separation is further enhanced by passing the probe through a "spatial filter" (10) consisting of a $50 \mu \mathrm{m}$ diameter pin hole located on a diaphragm (11) sandwiched between two focusing lenses. Finally, the probe beam is analyzed by an optical bridge detector composed of a polarizing beam splitter (12) and two photodiodes (13). The changes of the power and polarization acquired by the probe upon reflection from the sample provide information (primarily) about the pump induced dynamics in the electron-lattice and magnetic subsystems, respectively. In particular, the polarization signal results from the polar Kerr effect and is therefore proportional to the out-of-plane component of the magnetization. The sample is subjected to a fixed magnetic field equal to $\mathbf{H}_{\mathrm{ext}}=(-120 \mathbf{x}+50 \mathbf{y}+$ $470 z$ ) Oe at the sample position. Static magneto-optical hysteresis loop measurements have confirmed that the projection of the magnetic field onto the sample's $(X Y)$ plane exceeds the in-plane saturation field.

Figures 1(b)-1(j) display $10 \times 10 \mu \mathrm{m}^{2}$ Kerr images of the film surface at various time delays [27]. The images are centered at the point hit by the pump beam resulting in the dark spot. Despite the short duration of the pump pulse, the dark spot survives until the arrival of the next pump pulse (i.e., for $12.5 \mathrm{~ns}$ ). Figure 1(b) at the time delay of $0.667 \mathrm{~ns}$ ("negative time delay", i.e., $0.333 \mathrm{~ns}$ before the arrival of the pump pulse) shows a dark spot resulting from the incomplete relaxation of transients induced by the previous pump pulse [28]. Figure 1(c) shows the Kerr image at a time delay of $1.000 \mathrm{~ns}$, the moment when a new pump pulse strikes the sample resulting in the observed enhancement of the central dark spot, which is now accompanied by an adjacent smaller bright spot. Figure 1(d) shows the Kerr image at $1.200 \mathrm{~ns}$, by which time a dark $X$-shaped beam pattern develops around the dark or bright spot. The longer symmetry axis of the $X$ pattern is aligned roughly orthogonal to the in-plane projection of the applied field. Figures 1(e) and 1(f) show Kerr images at $1.267 \mathrm{~ns}$ and $1.367 \mathrm{~ns}$, respectively, and show the arms of the $X$ pattern moving away from the central dark spot with respect to Fig. 1(d). In addition, Fig. 1(f) reveals a circular ripple centered at the pump beam spot. At $1.450 \mathrm{~ns}$ [Fig. 1(g)], we observe strengthening of the circular ripple and further propagation of the $X$ pattern away from the center. Figures $1(\mathrm{~h})-1(\mathrm{j})$ display Kerr images at time delays of $1.567,1.733$, and $2.017 \mathrm{~ns}$, respectively. The circular ripple is observed to increase in diameter sequentially, while the $X$ pattern is expelled from the field of view.

To understand the $X$ pattern, we performed micromagnetic simulations of a $5 \times 5 \mu \mathrm{m}^{2}$ permalloy film of $50 \mathrm{~nm}$ thickness using MUMAX2 [29]. Periodic boundary conditions are used to suppress finite size effects expected for 
this sample size [30]. A saturation magnetization $M_{s}$ of $8 \times 10^{5} \mathrm{~A} / \mathrm{m}$, exchange stiffness $A$ of $1.3 \times 10^{-11} \mathrm{~J} / \mathrm{m}$, and zero magnetocrystalline anisotropy are assumed. As in the experiment, a field of $\mathbf{H}_{\mathrm{ext}}=(-120 \mathbf{x}+50 \mathbf{y}+$ $470 \mathbf{z})$ Oe is applied to slightly tip the magnetization out of the film plane. The simulations are based on the LandauLifshitz-Baryakhtar equation (LLBr) for the case of cubic crystalline symmetry [31]. The addition of the Baryakhtar relaxation to the Landau-Lifshitz torque equation enables description of the relaxation of the absolute value of the magnetization induced by ultrafast heating [2], along with much slower magnetization dynamics, i.e., precession. The process of the ultrafast demagnetization could itself be phenomenologically described by the LLBr equation. However, our model does not explicitly include coupling between the spin, lattice, and electron subsystems. So, to avoid speculations e.g., in the framework of the threetemperature model [1], the influence of the pump pulse on the sample is approximated by an instantaneous reduction of the saturation magnetization. Spatially, this reduction of the magnetization is represented by a combination of a Gaussian profile in the film plane (with FWHM of $800 \mathrm{~nm}$ ) and an exponential penetration of the optical excitation into the film (with a skin depth of $50 \mathrm{~nm}$ ), as shown in Fig. 2(a). This choice of parameters resembles the optical pump spot used in the experiment.

The recovery (relaxation) of the saturation magnetization with time is shown in Fig. 2(b) (red curve). The recovery is described by the $\mathrm{LLBr}$ relaxation term only, thereby assuming existence of a heat reservoir of infinite capacity to which the energy and angular momentum are channeled from the spin subsystem. From this point of view, our model predicts the minimum possible time for the magnetization recovery. As follows from the trivial solution of $\mathrm{LLBr}$, the recovery time is given by $\tau_{E}=$ $\chi /\left(\gamma \alpha M_{T=0}\right)$, where $\chi, \gamma, \alpha$, and $M_{T=0}$ are the longitudinal susceptibility, gyromagnetic ratio, Gilbert damping constant, and zero-temperature saturation magnetization, respectively. We make a rough assumption that these parameters are constant during the process of magnetization length recovery, and so, are independent of the temperature. The value of the recovery time obtained from fitting the (red) curve in Fig. 2(b) is found to be 113 fs, which agrees with the results of Radu et al. [32]. The localized reduction of the magnetization induced by the pump pulse creates a nonuniformity in the internal field that activates precession of magnetization around its local transient equilibrium orientation, as shown in Fig. 2(b) (green curve). Although the excitation can be considered to be broadband in the frequency domain, the relatively large spatial dimensions of the pump induced transients effectively limit the excitation to magnetostatic spin waves [33] with wavelengths of the order of the pump spot size $D$ and therefore wave vectors of the order of $k=\pi / D \approx 4 \times 10^{6} \mathrm{~m}^{-1}$. The dispersion of such spin waves is highly anisotropic, (a)

(c)
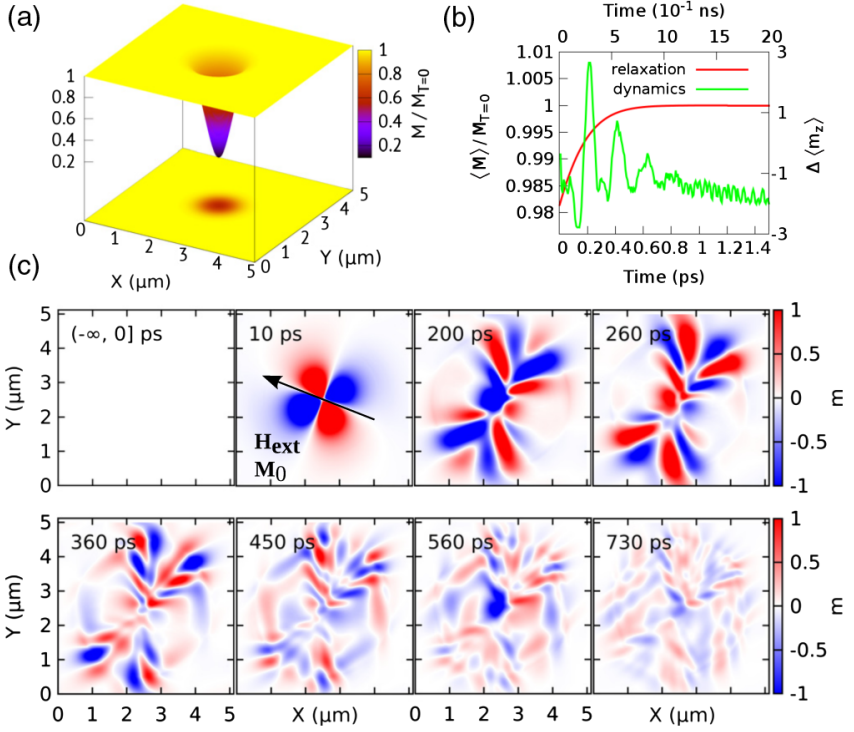

FIG. 2 (color online). (a) The initial Gaussian profile of the absolute value of the magnetization assumed in the simulations is shown. (b) The simulated recovery of the absolute value of the magnetization $\langle M\rangle$ averaged over the $5 \times 5 \mu \mathrm{m}^{2}$ sample is shown as a function of time (bottom axis) together with the net dynamic out-of-plane component of the magnetization $\Delta\left\langle m_{z}\right\rangle$ presented for longer time scales (top axis) for the epicenter of the pump pulse. (c) The simulated snapshots of the spatial profiles of the out-of-plane component of the magnetization are shown. The color contrast is given by $m=C\left(M_{z} / M_{s}-\right.$ $\left.M_{z 0} / M_{s 0}\right)$, where $C=100$ is a contrast adjustment constant and $M_{z 0}$ and $M_{s 0}$ are the equilibrium out-of-plane component and absolute value of the magnetization, respectively. $\mathbf{H}_{\text {ext }}$ and $\mathbf{M}_{0}$ denote the directions of the in-plane components of the applied static magnetic field and the initial magnetization, respectively.

in turn resulting in highly anisotropic propagation in the film plane, as shown in Fig. 2(c). The anisotropic dispersion dictates that the group and phase velocities of the spin waves are noncollinear. As a result, the waves propagate away from the pump spot in a "butterfly" fashion, which resembles the $X$ pattern observed in the experiment.

The spin wave pattern observed in Fig. 2(c) is neither symmetric nor antisymmetric relative to the direction of the in-plane projection of the bias field. A symmetric pattern would indicate that the excitation results from the transient out-of-plane demagnetizing field [14]. In contrast, the torque induced by a symmetric transient in-plane demagnetizing field [34] is actually antisymmetric, which would result in an antisymmetric spin wave emission pattern. The presence of both mechanisms produces the mixed pattern shown in Fig. 2(c).

To gain further insight into the observed pattern of spin wave propagation, we consider the $2 \mathrm{D}$ dispersion relation of magnetostatic spin waves in a continuous $50 \mathrm{~nm}$ thick film of Permalloy lying in the $x-y$ plane, with an external field of 150 Oe aligned along the $y$ direction [Fig. 3(a)]. The constant frequency lines 

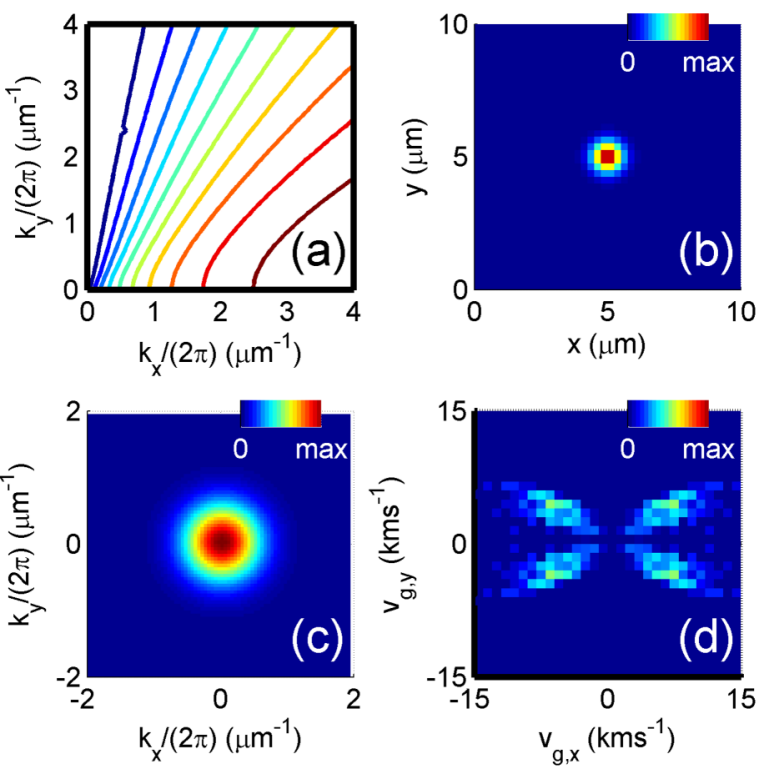

FIG. 3 (color online). (a) Constant frequency curves ranging from 4 to $13 \mathrm{GHz}$ (from left to right) are shown in steps of $1 \mathrm{GHz}$ for magnetostatic spin waves on a $50 \mathrm{~nm}$ thick permalloy film under a field of 150 Oe applied along the $y$ direction. (b) The spatial profile of the pump spot is shown on the sample surface of a $10 \times 10 \mu \mathrm{m}^{2}$ area. (c) The Fourier transform of the pump spot profile from (b) is shown in the reciprocal space. (d) The distribution of the efficiency of excitation of magnetostatic spin waves by the pump pulse from (b) and (c) is shown in the group velocity space.

(so-called "slowness curves") in this figure allow us to calculate the group velocity at every point in this reciprocal (wave vector) space. Figure 3(b) shows the spatial profile of the optical pump spot with the FWHM of $0.8 \mu \mathrm{m}$, and Fig. 3(c) shows the corresponding spatial Fourier transform spectrum in the reciprocal space. Figure 3(d) shows the "group velocity space," where the group velocity at every point in the wave vector space of Fig. 3(a) is calculated and binned with a weighting factor given by the Fourier amplitude shown in Fig. 3(c). As a result, an $X$ pattern with shape and orientation with respect to the applied field ( $y$ direction) that agree with the experiment is clearly observed in Fig. 3(d). This allows us to interpret the observed pattern as consisting of so-called spin wave caustic beams, reported in Refs. [18,35-38]. The major difference between this experimental study and those of other groups is that our experiment is broadband in both frequency and wave vector domains resulting from the extremely short time duration of the pump pulse and the tightly focused optical pump spot while the previous studies were performed at a single frequency of the microwave excitation.

Let us now discuss the propagating circular ripple observed experimentally but not in the micromagnetic simulations. The perfectly circular shape of the ripple and the lack of dependence on the applied magnetic field allow us to identify it as the previously observed SAWs [22]. The observed propagation speed of the ripple, as judged by the growth of the circular pattern diameter with time, roughly equals $5 \mathrm{~km} / \mathrm{s}$, which agrees reasonably with the value predicted for the Rayleigh SAW for typical Permalloy and sapphire elastic constants [39]. The SAW is observed in the Kerr signal due to its "pollution" by the parasitic reflectivity signal, in which the propagating ripple is also observed albeit less distinctly (therefore not shown). This artifact results from small misalignments in the experiment and is quite common in all-optical pump-probe measurements [28].

In conclusion, we have experimentally demonstrated a direct excitation of propagating spin waves of micrometer wavelength by ultrafast laser pulses via process of ultrafast demagnetization. The micromagnetic simulations performed with account of the longitudinal relaxation of the magnetization vector have reproduced the experimental observations, confirming the excitation mechanism and supporting the interpretation of the observed caustic pattern of spin wave propagation in terms of noncollinear magnonic group and phase velocities dictated by the anisotropic dispersion of magnetostatic spin waves in thin magnetic films. The development of the experimental (TROPSOM) and numerical (MUMAX2) tools, demonstrated in this study, will prove instrumental for further advances in the fields of magnonics and optomagnetism, as well as their crossing - photomagnonics.

The authors gratefully acknowledge the assistance of R. J. Hicken, T. Isaac, and R. Mikhaylovskiy in setting up the experiments, and thank S. Bailey and R. Sooryakumar for providing the calculation of the SAW velocity. The research leading to these results has received funding from the European Commission's 7th Framework Programme (FP7/2007-2013) under Grant Agreements No. 228673 (MAGNONICS) and No. 233552 (DYNAMAG), from the EPSRC of the United Kingdom under project EP/E055087/1, and from the Flanders Research Foundation (FWO).

[1] A. Kirilyuk, A. V. Kimel, and Th. Rasing, Rev. Mod. Phys. 82, 2731 (2010), and references therein.

[2] E. Beaurepaire, J.-C. Merle, A. Daunois, and J.-Y. Bigot, Phys. Rev. Lett. 76, 4250 (1996).

[3] A. V. Kimel, A. Kirilyuk, P. A. Usachev, R. V. Pisarev, A. M. Balbashov, and Th. Rasing, Nature (London) 435, 655 (2005).

[4] C.D. Stanciu, F. Hansteen, A. V. Kimel, A. Kirilyuk, A. Tsukamoto, A. Itoh, and Th. Rasing, Phys. Rev. Lett. 99, 047601 (2007).

[5] K. Vahaplar, A. M. Kalashnikova, A. V. Kimel, D. Hinzke, U. Nowak, R. Chantrell, A. Tsukamoto, A. Itoh, A. Kirilyuk, and Th. Rasing, Phys. Rev. Lett. 103, 117201 (2009).

[6] J.-Y. Bigot, M. Vomir, and E. Beaurepaire, Nat. Phys. 5, 515 (2009). 
[7] A. V. Kimel, B. A. Ivanov, R. V. Pisarev, P. A. Usachev, A. Kirilyuk, and Th. Rasing, Nat. Phys. 5, 727 (2009).

[8] B. Koopmans, G. Malinowski, F. D. Longa, D. Steiauf, M. Fähnle, T. Roth, M. Cinchetti, and M. Aeschlimann, Nat. Mater. 9, 259 (2010).

[9] N. Kanda, T. Higuchi, H. Shimizu, K. Konishi, K. Yoshioka, and M. Kuwata-Gonokami, Nat. Commun. 2, 362 (2011).

[10] I. Radu et al., Nature (London) 472, 205 (2011).

[11] K. Vahaplar, A. M. Kalashnikova, A. V. Kimel, S. Gerlach, D. Hinzke, U. Nowak, R. Chantrell, A. Tsukamoto, A. Itoh, A. Kirilyuk, and Th. Rasing, Phys. Rev. B 85, 104402 (2012).

[12] A. R. Khorsand, M. Savoini, A. Kirilyuk, A. V. Kimel, A. Tsukamoto, A. Itoh, and Th. Rasing, Phys. Rev. Lett. 108, 127205 (2012).

[13] T. A. Ostler et al., Nat. Commun. 3, 666 (2012).

[14] M. van Kampen, C. Jozsa, J. T. Kohlhepp, P. LeClair, L. Lagae, W. J. M. de Jonge, and B. Koopmans, Phys. Rev. Lett. 88, 227201 (2002).

[15] A. Barman, S. Wang, S. Wang, J. D. Maas, A. R. Hawkins, S. Kwon, A. Liddle, J. Bokor, and H. Schmidt, Nano Lett. 6, 2939 (2006).

[16] V. V. Kruglyak and R. J. Hicken, J. Magn. Magn. Mater. 306, 191 (2006).

[17] B. Lenk, G. Eilers, J. Hamrle, and M. Münzenberg, Phys. Rev. B 82, 134443 (2010).

[18] T. Satoh, Y. Terui, R. Moriya, B. A. Ivanov, K. Ando, E. Saitoh, T. Shimura, and K. Kuroda, Nat. Photonics 6, 662 (2012).

[19] V. V. Kruglyak, S. O. Demokritov, and D. Grundler, J. Phys. D 43, 264001 (2010), and references therein.

[20] B. Lenk, H. Ulrichs, F. Garbs, and M. Münzenberg, Phys. Rep. 507, 107 (2011), and references therein.

[21] A. A. Serga, A. V. Chumak, and B. Hillebrands, J. Phys. D 43, 264002 (2010), and references therein.

[22] Y. Sugawara, O. B. Wright, O. Matsuda, M. Takigahira, Y. Tanaka, S. Tamura, and V. E. Gusev, Phys. Rev. Lett. 88, 185504 (2002).

[23] H. Ulrichs, V.E. Demidov, S. O. Demokritov, and S. Urazhdin, Appl. Phys. Lett. 100, 162406 (2012).

[24] Y. Au, T. Davison, E. Ahmad, P. S. Keatley, R. J. Hicken, and V. V. Kruglyak, Appl. Phys. Lett. 98, 122506 (2011).
[25] Y. Au, E. Ahmad, O. Dmytriiev, M. Dvornik, T. Davison, and V. V. Kruglyak, Appl. Phys. Lett. 100, 182404 (2012).

[26] M. Dvornik, Y. Au, and V. V. Kruglyak, Top. Appl. Phys. 125, 101 (2013), and references therein.

[27] See Supplemental Material at http://link.aps.org/ supplemental/10.1103/PhysRevLett.110.097201 for Fig. 1 with enhanced contrast and for the TROPSOM data in a movie format.

[28] R. Wilks, N. D. Hughes, and R. J. Hicken, J. Appl. Phys. 91, 8670 (2002).

[29] A. Vansteenkiste and B. Van de Wiele, J. Magn. Magn. Mater. 323, 2585 (2011).

[30] A. Barman, V. V. Kruglyak, R. J. Hicken, J. M. Rowe, A. Kundrotaite, J. Scott, and M. Rahman, Phys. Rev. B 69, 174426 (2004).

[31] I. V. Baryakhtar and V. G. Baryakhtar, Ukr. J. Phys. 43, 1433 (1998).

[32] I. Radu, G. Woltersdorf, M. Kiessling, A. Melnikov, U. Bovensiepen, J. U. Thiele, and C. H. Back, Phys. Rev. Lett. 102, 117201 (2009).

[33] R. W. Damon and J. R. Eshbach, J. Phys. Chem. Solids 19, 308 (1961).

[34] See e.g., Fig. 3 in G. Eilers, M. Lüttic, and M. Münzenberg, Phys. Rev. B 74, 054411 (2006).

[35] V.E. Demidov, S. O. Demokritov, D. Birt, B. O'Gorman, M. Tsoi, and Xiaoqin Li, Phys. Rev. B 80, 014429 (2009).

[36] T. Schneider, A. A. Serga, A. V. Chumak, C. W. Sandweg, S. Trudel, S. Wolff, M. P. Kostylev, V.S. Tiberkevich, A. N. Slavin, and B. Hillebrands, Phys. Rev. Lett. 104, 197203 (2010).

[37] M. P. Kostylev, A. A. Serga, and B. Hillebrands, Phys. Rev. Lett. 106, 134101 (2011).

[38] V. Veerakumar and R. E. Camley, Phys. Rev. B 74, 214401 (2006).

[39] The calculation was done using the Green function approach from A. G. Every, K. Y. Kim, and A. A. Maznev, J. Acoust. Soc. Am. 102, 1346 (1997). For Permalloy, mass density $\rho=8.69 \mathrm{~g} \mathrm{~cm}^{-3}$ and elastic constants $c_{11}=$ $265 \mathrm{GPa}$ and $c_{44}=66 \mathrm{GPa}$ were assumed. For sapphire, we assumed $\rho=3.98 \mathrm{~g} \mathrm{~cm}^{-3}, c_{11}=496 \mathrm{GPa}$, and $c_{44}=$ $148 \mathrm{GPa}$. 